\title{
Mobile Phone-based Multimedia Application Could Improve Maternal Health in Rural Southwestern Uganda: Mixed Methods Study
}

\author{
Angella Musiimenta ${ }^{1 *}$, Wilson Tumuhimbise ${ }^{1}$, Godfrey Mugyenyi ${ }^{1}$, Jane Katusiime ${ }^{1}$, Esther C \\ Atukunda ${ }^{1}$, Niels Pinkwart ${ }^{2}$ \\ ${ }^{1}$ Mbarara University of Science and Technology, Mbarara, Uganda, ${ }^{2}$ Angels Compassion Research and \\ Development Centre (ACORD), Mbarara, Uganda, ${ }^{3}$ Humboldt-Universität zu Berlin
}

\begin{abstract}
Background: Reducing maternal and infant mortality rates remains challenging. Illiteracy, lack of reliable information, long distances to health centers continue to limit access to quality maternal healthcare in Uganda. Mobile health technologies could be promising affordable strategies for enhancing access to maternal health services. However, there is lack of studies assessing the experiences of illiterate rural pregnant women regarding these technologies.

Objective: To explore how illiterate pregnant women perceive a maternal health mobile application composed of tailored video and audio messages, appointment reminders and calling function.

Methods: We purposively sampled illiterate pregnant women initiating antenatal care at Mbarara Regional Referral Hospital. We carried out three focus group discussions with 14 women to elicit information on perceptions of the proposed mobile phone based multimedia application. We used STATA 13 to describe study participants and their preferences.

Results: Pregnant women anticipated that intervention would enhance maternal health by reminding them to attend antenatal appointments, enabling transport cost and time saving, providing tailored information that is easy to understand, and recall. However, financial constraints and phone sharing would limit the functionality.

Conclusion: Mhealth application may provide acceptable and affordable alternative approaches to providing maternal health services, especially in settings where face-to-face approaches are challenging.

Keywords: mobile health technologies, maternal health, Illiterate women, videos/audios, multimedia, appointment reminders.

Correspondence: * amusiimenta@must.ac.ug

DOI: 10.5210/ojphi.v12i1.10557

Copyright @2020 the author(s)

This is an Open Access article. Authors own copyright of their articles appearing in the Online Journal of Public Health Informatics. Readers may copy articles without permission of the copyright owner(s), as long as the author and OJPHI are acknowledged in the copy and the copy is used for educational, not-for-profit purposes.
\end{abstract}




\section{Introduction}

\section{Maternal Health}

Reducing maternal and infant mortality rates remains a global priority. In many Low Resource Countries (LRCs), maternal and child mortality rates remain unacceptably high. In 2013, 289,000 deaths occurred globally, of which 286,000 occurred in developing countries alone [1]. The maternal mortality rate in Uganda is estimated at 400 maternal deaths per 100,000 live births [2]. This rate is considered to be among the highest in the world, with maternal mortality being more likely for women living in rural areas and among poorer communities. Statistics about maternal health in Uganda should be interpreted with caution since $62 \%$ of women deliver outside the health facility without skilled care and thus their data is not captured. For every maternal death in Uganda, at least six women survive with chronic and debilitating ill health [3]. Although Uganda had previously registered a decline in infant and under-five mortality, recent statistics show that currently, 17 women die every day while 106 new-born babies die every day [4]. Most causes of these deaths are preventable maternal-related complications such as severe bleeding, infections, unsafe abortion, pregnancy-induced hypertension, obstructed labor, and lack of access to life saving HIV treatment [5]. The report further indicated that illiteracy, poverty, distance to health care centers, and cultural practices continue to limit access to quality health care in Uganda. Being pregnant in Uganda is nowadays believed to be 'putting one leg in the grave' as the chances of being alive after delivery are much lower than in other regions of the world. Only about 33\% of pregnant mothers receive the recommended four antenatal visits per partum [2]. Many women especially those from rural areas continue to lack reliable maternal health information - they rely on information from their peers which is mixed with cultural taboos, and is not accurate since pregnancy treats each woman differently. Access to essential health services and information, particularly for the vulnerable rural pregnant women becomes even more complicated given the current COVID-19 pandemic due to the overwhelmed healthcare systems.

\section{Potentials of mobile Health Technologies}

Traditional approaches to accessing health information from the health facilities may be limited by geographical boundaries, are often expensive due to the time and transport costs involved, and may be constrained by poor communication and records management, and chronic shortages of health professionals particularly in LRCs [6,7]. The use of Information Communication Technology (ICT)-based approaches to disseminating information such as multimedia mobile applications, websites, and social media can minimize the challenges associated with face-to-face approaches [8]. Rapidly expanding cellular networks across sub-Saharan Africa have greatly increased the capacity of cellular technology to serve as a novel solution to challenges and barriers to maternal health. According to Global System for Mobile Communications (GSMA) real-time intelligence data, there are currently over 5.13 billion people with mobile devices, and 8.97 billion mobile connections which exceeds the current world population of 7.71 billion. Moreover, in Uganda specifically, mobile phone reception is available across the vast majority of the country, including many rural areas where $80 \%$ of population lives of which majority are economically disadvantaged [9]. Given the widespread telephone ownership and mobile network coverage in sub-Saharan Africa- (76\% ownership in 2015 [10], mobile health-based interventions can potentially provide affordable means of providing reliable information to expectant mothers. Mobile phone infrastructure is being utilized widely in multiple sectors, such as micro-banking via 
services like Mobile Money and M-PESA, which has resulted in inclusive development in LRCs by unlocking opportunities to reach the otherwise underserved populations [11]. Such applications can potentially provide accessible and affordable means of providing reliable maternal health information to the otherwise hard-to-reach rural women.

\section{Mobile health Technologies for Maternal Health}

From our prior work, we have demonstrated that using ICTs such as networked and mobile telephones is acceptable and feasible, and useful in bringing services to patients regardless of geographical barriers and social denials, and can cater for privacy and confidentiality aspects [1214]. Although the application of mobile technologies in supporting maternal and child health is still rare especially in developing countries, they are promising tools for innovatively disseminating pregnant-related information to women [15]. While there are few attempts to improve maternal health in developing countries by use of mobile technology through innovations such as MomConnect and mobile moms [16], the need for more research on the use of mobile phones in maternal health is well documented in a systematic review [17].

\section{Mobile Health Technologies for Illiterate Women}

There are generally limited studies that have utilized mobile health intervention to support maternal health especially in LRCs. The modest existing studies report positive benefits; such as increased antenatal attendance as a result of maternal health-related SMS (Short Messaging Service) texts sent to pregnant women [16,18-20], and strengthened women's relationship with healthcare providers [21]. However, these studies have mainly utilized SMS texts, benefit women who can read and write while leaving out illiterate women who are, in most cases, more prone to maternal health problems. The use of biweekly voice messages in local language (with maternal and child health contents) which were sent to the mobile phone of pregnant women's spouses motivated mothers to visit antenatal postnatal clinics in India [22]. In Uganda, 33.7\% of women of 25 years and above have never been to school, while $41.2 \%$ only completed some primary level education [23]. Rural illiterate and semi-illiterate expectant women in Uganda continue to lack access to friendly, confidential, reliable, and interactive maternal health information - about what to expect, and what to do at different stages of pregnancy, good feeding habits, as well as information about the benefits of delivering from the hospital, which results in many delivering from their homes without a qualified birth attendant. Against this background, this study explored how pregnant women perceive a maternal health mobile application composed of tailored video and audio messages, appointment reminders and calling function.

\section{Methods}

\section{Study design and setting}

The study combines focus group discussions and surveys in a parallel mixed methods study design. Participants (pregnant mothers) were recruited from Mbarara Regional Referral Hospital (MRRH), which is the largest hospital in rural southwestern Uganda. The MRRH employs 11 obstetricians and 22 midwives and performs over 10,000 deliveries annually with a maternal mortality rate of $270 / 100,000$ live birth, caesarean section rate of $30 \%$ and a perinatal mortality rate of 56/1000 [24]. Sociodemographic and basic health data are captured from pregnant women during their first 
visit, and stored in paper-based antenatal registers. Each woman is given an antenatal card that contains her biodata as well as the date of the next appointment. Women are expected to attend at least four antenatal appointments, and they are supposed to bring their antenatal cards on every visit. The clinic verbally provides group-based health talks to women. The talks are scheduled according the trimesters - first trimester talks are offered on Tuesdays, second trimester talks are offered on Wednesdays, while third trimester health talks are offered on Thursdays.

Topics covered in these talks include nutrition and birth preparedness. There are currently no follow-up mechanism for pregnant women who miss attending their antenatal appointments.

\section{Selection of study participants}

Between October 2019 and February 2020, we purposively sampled pregnant women receiving antenatal care from the department of obstetrics and gynaecology, MRRH. Inclusion criteria were as follows: a) initiating antenatal care at MRRH at the earliest presentation in the first and second trimester, b) being illiterate (not having studied beyond primary seven or elementary education), c) 18 years and above, d) residents of Mbarara (within $20 \mathrm{~km}$ of MRRH) e) willing and able to give informed consent.

\section{The Mobile Phone-based Multimedia Application for Maternal health}

We developed the multimedia mobile health application using Java programming language, while the database that hosts multimedia messages was developed using SQlite. It is an offline (standalone) application which was developed following user-centered design approaches that involved incorporating input from pregnant women as prospective users. The application was installed on affordable smart phones provided by the study. Pregnant women were provided with solar chargers to supplement electricity charging. The application has three major functionalities;

1. The video/audio function; which provides locally customized videos and audios that provides personalized maternal health information to pregnant women based on their pregnancy stages. Contents of the videos/audio messages include; nutrition, breast feeding, HIV testing, spouse involvement, family planning, danger signs, preparing for child birth, care during pregnancy, care during delivery and postnatal care.

2. The appointment reminder function allows the users to set the dates and reminders for their next antenatal care appointment.

3. The calling function through which the pregnant women can communicate with health workers and ask pregnancy-related queries or application related issues.

It also has login module which uses pictorial password to allow access to the application.

\section{Study procedures}

Before the focus group discussion, we first oriented each participant about the potential mobile application to support maternal health. First, we explained and demonstrated how the application works including how to login to the application, view the multimedia videos, audios, set antenatal appointment reminders, and call the specialist in case of an inquiry. Participants were then given 
the application and asked to explain what it does and to practically demonstrate how it works to the researchers.

\section{Data collection}

From a private space at a research office near the MRRH, AM, WT, and GM carried out three focus group discussions with illiterate pregnant mothers (totaling to 14). Each focus group discussion lasted between 40 and 60 minutes. All questions in the focus group guide were translated into the local language (Runyankole) and back-translated to English by a different translator, after which the two versions were compared for accuracy. The focus group discussion were carried out in Runyankole (local language). The discussions were digitally recorded, transcribed, and translated to English. We elicited participants' opinions about the usage of a mobile phone-application to support maternal health, including anticipated benefits and challenges of using the intervention. Following each interview, NP and JK reviewed transcripts for quality, clarity, and detail. TW and AM also administered surveys to pregnant women to collect information on socio-demographics, socio economic status, food security, HIV status, and cellular phone ownership and utilization.

\section{Analysis}

We used inductive content analysis [25] to derive categories describing and summarizing how participants perceived the intervention. Initially, AM and WT reviewed and discussed $20 \%$ of transcripts for content relevant to participants' perceptions about the intervention, anticipated benefits, and challenges. AM and WT then assembled a codebook from the identified concepts, using an iterative process, which included developing codes to represent content, writing operational definitions, and selecting illustrative quotes. NP, JK, and GM also reviewed and discussed the codebook. Following completion of the codebook, AM and WT applied codes using NVIVO 11. Differences in coding were harmonized through discussion. AM and TW used STATA 13 to describe study participants' characteristic, their socio economic status, food security, intervention preference, pregnant-related information, and technology readiness.

\section{Human subjects and ethics approval}

All participants provided signed informed consent before study participation. The Institutional Review Committee of Mbarara University of Science and Technology, the Uganda National Council for Science and Technology, approved this study.

\section{Results}

\section{Survey Results}

Participant characteristics 
Table 1: Socio-demographic and basic health status characteristics of study participants

\begin{tabular}{|l|l|}
\hline & \multicolumn{1}{|c|}{$\begin{array}{c}\text { Pregnant women } \\
(\mathbf{n = 1 4}(\mathbf{\%}))\end{array}$} \\
\hline Median age (years) include IQR & $26.00(\mathrm{IQR}=11)$ \\
\hline Marital status & Married 11(78.6) \\
\hline Highest level of education & \\
\hline P1- P7 & $11(78.6)$ \\
\hline No education & $3(21.4)$ \\
\hline Able to read English & $4(28.6)$ \\
\hline Able to read Runyankole & $11(78.6)$ \\
\hline Living with HIV & $4(28.6)$ \\
\hline Mean (SD) months of pregnancy & $3.93(1.730)$ \\
\hline
\end{tabular}

$\mathrm{SD}=$ standard deviation

Of the 23 screened pregnant women, 9 (39.13\%) were excluded. Pregnant women were excluded for (individuals could have $>1$ criterion): unwillingness to give consent $(n=3 ; 33.33 \%)$, lived beyond $20 \mathrm{~km}$ from MRRH $(\mathrm{n}=4,44.44 \%)$, below 18 years $(\mathrm{n}=1,11.11 \%)$ and studied beyond primary seven $(n=1,11.12 \%)$. A total of 14 pregnant women, of whom $4(28.6 \%)$ were persons living with HIV/AIDS, enrolled in the study between October 2018 and February 2019 (table 1). The majority of participants had not gone beyond primary seven $(n=11 ; 78.6 \%)$ and were able to read Runyankole (local language) $(\mathrm{n}=11 ; 78.6 \%)$.

Table 2: Current pregnancy-related information

\begin{tabular}{|c|c|}
\hline & $\begin{array}{c}\text { Pregnant women } \\
\qquad(n=14)\end{array}$ \\
\hline \multicolumn{2}{|c|}{ Current source of information about pregnant* } \\
\hline Friends and relatives & $6(42.9)$ \\
\hline Health care provider & $11(78.6)$ \\
\hline Internet & $2(14.3)$ \\
\hline Mass media e.g. radio/television & $2(14.3)$ \\
\hline \multicolumn{2}{|c|}{ Frequency of getting information about pregnant* } \\
\hline Per month & $11(78.6)$ \\
\hline More than a month & $3(21.4)$ \\
\hline \multicolumn{2}{|c|}{ Form of information about pregnant received* } \\
\hline
\end{tabular}




\begin{tabular}{|l|l|}
\hline Word of mouth & $13(92.9)$ \\
\hline Written text & $4(28.6)$ \\
\hline Video/audio & $5(35.7)$ \\
\hline Pregnancy information often got* & \\
\hline Nutrition & $11(78.6)$ \\
\hline Antenatal check-up reminders & $10(71.4)$ \\
\hline HIV testing & $9(64.3)$ \\
\hline Birth preparedness & $11(78.6)$ \\
\hline Danger signs & $11(78.6)$ \\
\hline Exercises & $9(64.3)$ \\
\hline Using a mosquito net & $1(12.5)$ \\
\hline
\end{tabular}

As indicated in table 2 above, the majority of pregnant women reported getting pregnancy-related information from healthcare providers $(n=11,78.6 \%)$, on a monthly basis $(n=11,78.6 \%)$, and by word of mouth $(\mathrm{n}=13,92.9 \%)$. Pregnant women often get information about nutrition $(\mathrm{n}=11$, $78.6 \%)$, birth preparedness $(n=11,78.6 \%)$, and danger signs $(n=11,78.6 \%)$.

Table 3: Technology Readiness

\begin{tabular}{|c|c|}
\hline & $\begin{array}{c}\text { Pregnant women } \\
(n=14)\end{array}$ \\
\hline \multicolumn{2}{|l|}{ Personal phone ownership } \\
\hline Yes & $12(85.7)$ \\
\hline No & $2(14.3)$ \\
\hline \multicolumn{2}{|l|}{ Type of phone } \\
\hline Feature phone & $12(85.7)$ \\
\hline Smart phone & $2(14.3)$ \\
\hline \multicolumn{2}{|l|}{ Phone sharing } \\
\hline Yes & $5(35.7)$ \\
\hline No & $9(64.3)$ \\
\hline \multicolumn{2}{|l|}{ Ever received pregnancy information on phone } \\
\hline Yes & $2(14.3)$ \\
\hline No & $12(85.7)$ \\
\hline $\begin{array}{l}\text { Preferred frequency of receiving information about pregnancy on } \\
\text { phone }\end{array}$ & \\
\hline
\end{tabular}




\begin{tabular}{|l|l|}
\hline Daily & $6(42.9)$ \\
\hline Weekly & $7(50.0)$ \\
\hline Monthly & $1(7.1)$ \\
\hline Preferred content of information to be sent on phone & \\
\hline Antenatal check-up reminders & $12(85.7)$ \\
\hline Nutrition & $10(71.4)$ \\
\hline Birth preparedness & $12(85.7)$ \\
\hline Danger signs & $9(64.3)$ \\
\hline Breast feeding & $8(57.1)$ \\
\hline Immunization & $7(50.0)$ \\
\hline Preferred form of receiving information to be received on phone & \\
\hline Video & $11(78.6)$ \\
\hline Audio & $10(71.4)$ \\
\hline Images/still photos & $5(35.7)$ \\
\hline Text & $4(28.6)$ \\
\hline Phone calls & $1(7.1)$ \\
\hline Preferred length of the message & \\
\hline 2 to 4 minutes & $1(7.1)$ \\
\hline 5 minutes & $5(35.7)$ \\
\hline Above 5 minutes & $8(57.1)$ \\
\hline
\end{tabular}

As indicated in table 3 above, the majority of pregnant women owned mobile phones $(n=12$, $85.7 \%$ ), all of which were feature phones. Only $5(35 \%)$ reported sharing their mobile phones. The majority $(n=12,85.7 \%)$ had never used their phones to receive pregnant-related information. All of participants preferred receiving tailored maternal health-related information on mobile phones in local language (Runyankole). The most preferred form of messages was video $(\mathrm{n}=11,78.6 \%)$, followed by audio $(n=10,71.4 \%)$. Weekly messages $(n=7,50 \%)$ were preferred to daily $(n=6$, $42.9 \%$ ), while monthly messages were the least preferred $(\mathrm{n}=1,7.1 \%)$. Most participants preferred receiving messages in form of antenatal appointment reminders $(\mathrm{n}=12,85.7 \%)$, and birth preparedness $(\mathrm{n}=12,85.7 \%)$.

\section{Interview Results}

As explained below, participants (pregnant women) perceived that the intervention would improve maternal health through: 1) providing appointment reminders, 2) enabling transport cost and time saving, 3) source of friendly, tailored, information that is easy to understand, and recall, 4) enhancing male support and involvement, and 5) enhancing feelings of being cared for, and 
prompting self-care. All participants preferred receiving tailored maternal health-related information on mobile phones in local language (Runyankole). This includes information about antenatal checkup appointments reminders nutrition, birth preparedness, danger signs, breastfeeding, and immunization. Participants felt the functionality of the mobile application intervention may be limited by lack of phone ownership, sharing of phones, and financial constraints.

\section{Providing appointment reminders}

Pregnant women reported that using mobile phone-based application could remind them to attend their antenatal appointments by minimizing the possibilities of forgetting.

Receiving the information on a phone is better because it reminds you in case you have forgotten the next visit". It is very easy to forget your next appointment, but when the message comes, it reminds you.

\section{Enabling transport cost and time saving}

Pregnant mothers felt that receiving maternal health-related information on their mobile phones could save them transport costs that would otherwise be incurred in travelling to the hospital to access such information from healthcare providers. This is important since some of them reported walking long distances to the clinic for antenatal checkups, which sometimes causes them to miss some appointments especially in times when they are feel too weak to walk.

Since I can receive the information from my phone, it saves me money that I should have spent in form of transport to the clinic. It is not easy to get transport since I am not working, and sometimes I have to walk a very long distance to come here. Sometimes I fail to come when I have no transport, and I have no energy to walk. It also saves me time too, since I don't have to prepare and travel to the seminar room to get that information.

\section{Source of friendly, tailored, information that is easy to understand, and recall.}

Pregnant women narrated how the videos are being played by happy people compared to the unfriendly and tired faces that they often encounter at the clinic. They also reported that the videos and audios contained information they could easily understand since they are visual messages played in their local language, and can be replayed and listened to, as many times as wanted. This approach was preferred compared to the current approach used by the clinic that involves providing verbal general health talks which are not necessarily tailored to their specific needs.

Sometimes you show up at the clinic, and the doctors and nurses welcome you with gloomy tired faces and speak rudely to you, and they make you wait for a long time before they can attend to you, which even makes you fear to return. But I like these videos because the people in them are happy and speak kindly. Also, one can easily 
forget the verbal information from the clinic, but with these videos, I can see them now and again until I have mastered them, and can also refer to them in future, and even share them with my colleagues who are pregnant.

\section{Enhancing spouse support and involvement}

Pregnant women reported that receiving maternal-health related information on a mobile application could provide opportunities for them to share the information with their spouses. They narrated that this sharing would demonstrate to their spouses the importance of the contents of the videos/audios such as nutrition, and attending antenatal appointments. The women reported that this would motivate spouses to provide the required nutritional/transport support, which they would other be reluctant to provide.

When you receive information about nutrition on phone and you show that message to your husband that will encourage him to buy you the necessary food without bias.

It will be easier for me to get permission from my husband to come for checkup when I show him the reminder on phone. He will know that the hospital seriously wants me to attend, so he will give me transport and allow me to come, otherwise sometimes he tells me that I should not come especially when he has no money.

\section{Enhancing feelings of being cared for, and prompting self-care}

Pregnant women associated receiving maternal health information on phone with feelings of being cared for. Being seen using the application could be interpreted as having special people who care about their pregnancy. This perceived care could motivate other women to access maternal healthcare from hospitals.

I would be glad to receive the information since it can make me feel cared for. Even when other people see me using the application, they know that I have special people who are caring about my pregnancy so that when they become pregnant they feel convinced to go and get the same services from the hospital.

Women reported that receiving mobile phone-based information about their pregnancy would enhance self-pregnancy care including taking the right foods, and nutritional supplements such as iron and folic tablets. They narrated how they would take an active role in their health by complying with the information contained in the application.

It will enable us to know the right foods to eat during pregnancy, as well the medicines we have to take. For example, I have just heard about the need to take folic acid in the video; this is something I had never heard about before, so I am going to see how I can start taking it. 
Participants felt the functionality of the intervention may be limited by financial constraints and sharing of phones.

\section{Financial constraints may limit the effectiveness of the intervention}

Pregnant women anticipated instances when they could receive the information but fail to respond to it due to lack of money. One woman cited a possibility of receiving nutritional information when she has no money to buy the recommended food.

The problem comes in when you receive the information about nutrition yet you don't have money to buy the recommended food.

\section{Lack of personal phone ownership may constrain receipt of information}

Pregnant women who share their phones or have no phones perceived the possibility of not receiving the information sent via the intervention.

Sometimes my husband uses my phone, so when you send me a message indicating the foods I need to eat, he will deny having seen the message when he has no money to buy the food, because he knows that when I see the message, I will ask him to buy those foods.

\section{Discussion}

In this study, pregnant women reported that the multimedia mobile application for maternal health could support maternal health by reminding patients attend their antenatal appointments, which addresses forgetfulness that sometimes hinders adherence to antenatal visits. Receiving videos and audios with maternal health-related information, and the opportunity to call in when need be could relieve pregnant women of the transport burdens and time that they spend travelling or sometimes walking long distances to the clinics for the same services. The application was perceived as a source of friendly, and tailored maternal health information that, given its video/audio nature is easy to understand and recall compared to verbal health talks that women often get from the clinic. Accessing the videos/audio could provide an opportunity to share the information with spouses as a way of soliciting the support needed to buy the necessary foods and obtain transport to the clinic. Pregnant women perceived receiving videos and audios as being cared for by people who mind about their health. The knowledge obtained from videos and audios, and from calling specialists could be used by pregnant women to take an active role in improving their maternal health including taking the right foods, birth preparedness, identifying danger signs, as well as adopting breastfeeding and immunization. However, pregnant women felt the functionality of the mobile application intervention may be limited by financial constraints, and sharing of phones.

Reminding pregnant women to attend antennal visits through automatic appointment reminders could potentially reduce missed appointments as a result of forgetting the appointment date. Attending antenatal care from the clinic provides an opportunity to educate women about danger signs of pregnancy complication, empowers women to develop a birth plan, enables monitoring of the pregnancy, and reduces morbidity and mortality risks for the mother and the child [26]. According the Uganda Demographic and Health Survey 2016, only 58\% of rural women attend 
the recommended four antenatal visits compared to $65 \%$ of women from urban areas. This report continues to demonstrate that only $53 \%$ of women with no education attended the recommended for antenatal visits compared to $72 \%$ of women with more than a secondary education. Though not meant for illiterates, a recent systematic review demonstrates some evidence that mobile phone-based SMS text reminders can improve adherence to attending antenatal visits [27] in a low resource setting. Being reminded by mobile phone-SMS based reminders could potentially result into forming a habit of adopting a health behavior [28]. When pregnant women turn up on their scheduled appointments, they are likely to maximize their contact and communication with health care professionals. Forgetting to attend an antenatal appointment requires rescheduling a missed appointment, which is challenging given the prevailing heavy workloads of health care professionals, as well as the already overburdened healthcare systems in Uganda. Due to fear of being blamed by health care professionals for having missed antenatal appointments, some pregnant women consequently resort to not returning to the clinic and end up being lost followups, or resort to traditional birth attendants, or only turn up again at their delivery time, which can potentially put the health of a mother and her baby at risk.

The present study indicates that providing maternal health-related information through mobile phones could relieve the burden of transport costs, time commitments, as well as the hassle of having to walk for long distances to access this information from the clinics. Evidence demonstrates that lack of transport to antenatal facilities, and walking long distances constrain access and utilization of antenatal care especially among the poor and remote communities [29]. Related studies indicate that using mobile phone-based consultations by village health teams to support maternal health saved mothers unnecessary transportation costs and improved their attitudes towards adapting recommended maternal and child care practices [30].

Compared to the verbal health talks that pregnant women often get from the clinic, the mobile phone-based application was perceived to be a source of friendly and tailored maternal health information that can easily be understood and recalled. Weekly videos with a length of at least five minutes were preferred to monthly videos. Watching friendly faces in the videos was preferred to the often unfriendly physical faces that pregnant women encounter from the overworked health care providers. Lack of friendly maternal health services have previously been cited as a major barrier to adoption of maternal healthcare seeking behavior in low resource settings [31]. Providing tailored information could potentially increase women's knowledge, understanding, and engagement while at the same time creating a sense of empowerment to cope with pregnancy, which can prevent adverse maternal and child health outcomes [32]. Since they can be watched, paused, and repeated anytime, the videos and audios provide an opportunity for self-paced learning which facilitates learning and recall, that may otherwise not be possible with face-to-face approaches.

The mobile health application provides opportunities for pregnant women to share the videos, audios, and appointment reminders with their spouses which could motivate their spouses to support them and be involved in their maternal health. This support could be in form of transport/permission to the clinic for antenatal services and delivery, assistance with house chores, and nutritional and feeding support. Spouse support has been previously associated with utilization skilled birth services in the same setting [33]. Using mobile phone-based SMS texts though not necessarily with illiterate women has previously been associated with improved spouse support which consequently has positive impacts on utilization of maternal health services [34]. 
Pregnant women associated receiving videos and audios with feelings of being cared for by people who mind about their health. This could create a sense of connectedness to health care providers which could motivate women to comply with the demands of the application including attending clinic appointments in order not to disappoint the health care providers. This emotional support could provide women with reassurance, acceptance and encouragement to utilize maternal health services, while at the same helping them overcome pregnancy-related stress emanating from long waiting queues at the clinic, that are often exhausting, frustrating, and time-consuming [35]. The maternal health knowledge gained through watching/listening to the videos/audios could enhance self-care including taking the right foods, preparing for birth, identifying danger signs, as well as adopting best practices such as breastfeeding and immunization. Enhancing self-care through mobile phone-based technologies is useful in improving maternal health for example; it reduces still birth [36], facilitates healthy gestation weight gain [37], and encourages smoking caseation among pregnant women [38].

Some pregnant women reported that functionality of the mobile application may be limited by financial constraints particularly in an instance when the video about nutrition highlights foods that they cannot afford to purchase. Another constraint was that of sharing of mobile phones which may constrain the receipt of some information. The problem of phone sharing may not be significant since according the survey results, only a few participants shared their phones. However, given the multimedia nature of the application, it can only run on smart phones which were only owned by a couple of women.

The main strength of this study is that it used a mixed-methods approach to collect both closedand open-ended data. In addition, because the study was conducted in a rural sub-Saharan African area and population, it has implications for similar settings. This study also identifies important insights from pregnant women that can inform the development of mobile phone-based application for improving maternal health.

This study is limited by the fact that we asked participants about perceptions before they could use the intervention in real life. Although we practically demonstrated the use of the intervention to all participants, participants were not able to describe actual experiences using the application (videos, audios, appointment reminders, and calling function) as part of their daily routine. Lastly, findings could be vulnerable to social desirability bias because participants self-reported their preferences and anticipated uses of the application; moreover, before the researchers and application developers.

In sum, mobile health application could potentially provide useful, accessible, friendly and tailored approaches to improving maternal health. They can provide a promising alternative or complementary approach to providing maternal health information especially among the rural illiterate women who might not easily access the information from health facilities. As mobile health technologies proliferate, understanding how they are perceived by prospective users is critical to developing interventions that are acceptable, feasible and effective in improving maternal health. Findings from this formative study informed the development of a mobile health intervention that is being implemented in a randomized controlled trial (NCT04089800) whose primary outcome measure is adherence to antenatal visits. Secondary outcome measure include adherence to postnatal visits, number of women delivering from hospital, number of maternal complications, missed abortions, premature, number of maternal and newborn deaths ad maternal 
health-related knowledge, attitudes. Further research is needed to understand how participants in diverse settings perceive this intervention.

\section{Acknowledgment}

The study was funded by a grant from the German Ministry of Education and Research, under the German-African Innovation Incentive Award (01DG18004). AM is also supported by Fogarty International Center of the National Institutes of Health (K43TW010388), and SPIDER (Swedish Programme for ICT in Developing and Emerging Regions) for funding (Dnr: SU - 323 - 5.1.2$0419-17)$

\section{Reference}

1. World Health Organization. UNICEF. Trends in maternal mortality: 1990 to 2013: estimates by WHO, UNICEF, UNFPA, The World Bank and the United Nations Population Division. 2014.

2. WHO. Maternal mortality ratio data by country. WHO library (2015). 2015.

3. Uganda Ministry of Health (MOH), 2008. Road Map for Accelerating the Reduction of Maternal and Neonatal Mortality and Morbidity in Uganda 2006-2015.

4. Save_the_Children_Uganda. Childbirth in Uganda: Stories from women and health workers 2015 [cited 201921 st August]. Available from:

https://www.healthynewbornnetwork.org/hnn-content/uploads/Human-interest-storiesbooklet_final.pdf.

5. UNICEF. UNICEF Uganda Annual Report 2016. Supporting the Government to reach the most marginalized and deprived children and women across the country. [Accessed 21 August 2019]. 2016 [cited 201921 st August ]. Available from:

https://www.unicef.org/uganda/reports/unicef-uganda-annual-report-2016.

6. Musiimenta A. 2012. Information Technology-Mediated Issues in Sexual Health and HIV/AIDS Education. Electron J Inf Syst Dev Ctries. 53(3).

7. Musiimenta A. 2012. Contextual Mediators influencing the Effectiveness of Behavioural Change Interventions: A Case of HIV/AIDS Prevention Behaviours. Online J Public Health Inform. 4(2). doi:https://doi.org/10.5210/ojphi.v4i2.3988. PubMed

8. Musiimenta A, Tumuhimbise W, Nankunda M, Bangumya E, Atuhaire J, et al. 2019. Electronic Learning May Improve the Teaching and Learning of Mathematics and Science in Marginalized Schools in Nakivale Refugee Settlement, Uganda: A Baseline Analysis. Journal of Education and Development. 3(2), 63. https://doi.org/10.20849/jed.v3i2.611

9. NITA_U. National InformationTechnology Survey2017/18 Report. 2018.

10. Be Healthy ITU. Be Mobile Annual Report 2016. International Telecommunication Union. 2016. 
11. Asongu S, Boateng A. Introduction to special issue: mobile technologies and inclusive development in Africa. Taylor \& Francis; 2018.

12. Musiimenta A, Tumuhimbise W, Mugaba AT, Muzoora C, Armstrong-Hough M, et al. 2019. Digital monitoring technologies could enhance tuberculosis medication adherence in Uganda: Mixed methods study. J Clin Tuberc Other Mycobact Dis. 17, 100119. PubMed https://doi.org/10.1016/j.jctube.2019.100119

13. Musiimenta A, Atukunda EC, Tumuhimbise W, Pisarski EE, Tam M, et al. 2018. Acceptability and Feasibility of Real-Time Antiretroviral Therapy Adherence Interventions in Rural Uganda: Mixed-Method Pilot Randomized Controlled Trial. JMIR Mhealth Uhealth. 6(5). PubMed https://doi.org/10.2196/mhealth.9031

14. Katusiime J, Pinkwart N, eds. Supporting Maternal Health Education in Developing Countries Using Mobile Phones-Results of a Pilot Study. Proceedings of the First African Conference on Human Computer Interaction; 2016: ACM.

15. Touche D. Empowering and enabling decision makers to include mHealth services into budgets. Maternal messaging mHealth programmes. 2014.

16. Thompson S, Harrison M, A MM. Mobile- Ligalnan: Improving quality and access to maternal care in Timor- Leste. Health Alliance International report. 2013.

17. Sondaal SFV, Browne JL, Amoakoh-Coleman M, Borgstein A, Miltenburg AS, et al. 2016. Assessing the effect of mHealth interventions in improving maternal and neonatal care in lowand middle-income countries: a systematic review. PLoS One. 11(5), e0154664. PubMed https://doi.org/10.1371/journal.pone.0154664

18. Noordam AC, Kuepper BM, Stekelenburg J, Milen A. 2011. Improvement of maternal health services through the use of mobile phones. Trop Med Int Health. 16(5), 622-26. PubMed https://doi.org/10.1111/j.1365-3156.2011.02747.x

19. Entsieh AA, Emmelin M, Pettersson KO. 2015. Learning the ABCs of pregnancy and newborn care through mobile technology. Glob Health Action. 8(1), 29340. PubMed https://doi.org/10.3402/gha.v8.29340

20. Atnafu A, Otto K, Herbst $\mathrm{CH}$. 2017. The role of mHealth intervention on maternal and child health service delivery: findings from a randomized controlled field trial in rural Ethiopia. mHealth. 3(9). PubMed

21. McBride, B., O’Neil, J.D., Hue, T.T., Eni, R., Nguyen, C.V. and Nguyen, L.T., 2018. Improving health equity for ethnic minority women in Thai Nguyen, Vietnam: qualitative results from an mHealth intervention targeting maternal and infant health service access. Journal of Public Health, 2018, 40(suppl_2), pp.ii32-ii41.

22. Hazra A, Khan ME, Mondal SK. 2018. Mobile phone messaging to husbands to improve maternal and child health behavior in India. J Health Commun. 23(6), 542-49. PubMed https://doi.org/10.1080/10810730.2018.1483444 
23. UBOS. Uganda Demographic and Health Survey 2016. 2016.

24. Mugyenyi GR, Atukunda EC, Ngonzi J, Boatin A, Wylie BJ, et al. 2017. Functionality and acceptability of a wireless fetal heart rate monitoring device in term pregnant women in rural southwestern Uganda. BMC Pregnancy Childbirth. 17(1), 178. PubMed https://doi.org/10.1186/s12884-017-1361-1

25. Hsieh H-F, Shannon SE. 2005. Three approaches to qualitative content analysis. Qual Health Res. 15(9), 1277-88. PubMed https://doi.org/10.1177/1049732305276687

26. Serbanescu F, Goodwin MM, Binzen S, Morof D, Asiimwe AR, et al. 2019. Addressing the First Delay in Saving Mothers, Giving Life Districts in Uganda and Zambia: Approaches and Results for Increasing Demand for Facility Delivery Services. Glob Health Sci Pract. 7(Supplement 1), S48-67. PubMed https://doi.org/10.9745/GHSP-D-18-00343

27. Wagnew F, Dessie G, Alebel A, Mulugeta H, Belay YA, et al. 2018. Does short message service improve focused antenatal care visit and skilled birth attendance? A systematic review and meta-analysis of randomized clinical trials. Reprod Health. 15(1), 191. PubMed https://doi.org/10.1186/s12978-018-0635-z

28. Musiimenta A, Atukunda EC, Tumuhimbise W, Haberer JE. Resilience after withdrawing a technology-based medication adherence support intervention from people living with HIV in rural Uganda. AIDS care. 2018;30(sup5):S89-S96.

29. Wilunda C, Scanagatta C, Putoto G, Montalbetti F, Segafredo G, et al. 2017. Barriers to utilisation of antenatal care services in South Sudan: a qualitative study in Rumbek North County. Reprod Health. 14(1), 65. PubMed https://doi.org/10.1186/s12978-017-0327-0

30. Mangwi Ayiasi R, Atuyambe LM, Kiguli J, Orach CG, Kolsteren P, et al. 2015. Use of mobile phone consultations during home visits by Community Health Workers for maternal and newborn care: community experiences from Masindi and Kiryandongo districts, Uganda. BMC Public Health. 15(1), 560. PubMed https://doi.org/10.1186/s12889-015-1939-3

31. Shahabuddin A, Delvaux T, Nöstlinger C, Sarker M, Bardají A, et al. 2019. Maternal health care-seeking behaviour of married adolescent girls: A prospective qualitative study in Banke District, Nepal. PLoS One. 14(6), e0217968. PubMed https://doi.org/10.1371/journal.pone.0217968

32. Lagendijk J, Been J, Ernst-Smelt H, Bonsel G, Bertens L, et al. 2019. Client-tailored maternity care to increase maternal empowerment: cluster randomized controlled trial protocol; the healthy pregnancy 4 All-2 program. BMC Pregnancy Childbirth. 19(1), 4. PubMed https://doi.org/10.1186/s12884-018-2155-9

33. Clement M, Mirembe ME, Deus T, Archbald B. 2019. Socio-Demographic and Maternal Determinants of Birth Preparedness among Pregnant Women Seeking Skilled Birth Services at a Rural General Hospital, Mid Western Uganda. Open J Nurs. 9(1), 68-77. https://doi.org/10.4236/ojn.2019.91007 
34. Ilozumba O, Dieleman M, Van Belle S, Mukuru M, Bardají A, et al. 2018. Multistakeholder perspectives on maternal text messaging intervention in Uganda: Qualitative study. JMIR Mhealth Uhealth. 6(5), e119. PubMed https://doi.org/10.2196/mhealth.9565

35. Nisar YB, Aurangzeb B, Dibley MJ, Alam A. 2016. Qualitative exploration of facilitating factors and barriers to use of antenatal care services by pregnant women in urban and rural settings in Pakistan. BMC Pregnancy Childbirth. 16(1), 42. PubMed https://doi.org/10.1186/s12884-016-0829-8

36. Weller M, Gardener G, Henry S, Ellwood D, Daly L, et al. 2018. My Baby’s Movements: Integration of a mobile phone application into the antenatal education toolkit. Women Birth. 31, S11. https://doi.org/10.1016/j.wombi.2018.08.040

37. Dahl AA, Dunn CG, Boutté AK, Crimarco A, Turner-McGrievy G. 2018. Mobilizing mHealth for moms: A review of mobile apps for tracking gestational weight gain. Journal of Technology in Behavioral Science. 3(1), 32-40. https://doi.org/10.1007/s41347-017-0030-6

38. Heminger CL, Schindler-Ruwisch JM, Abroms LC. 2016. Smoking cessation support for pregnant women: role of mobile technology. Subst Abuse Rehabil. 7, 15. PubMed https://doi.org/10.2147/SAR.S84239 